handelt man nur Personen mit einem Zehnjahres-Risiko von über 20 Prozent, sinkt die NNT in einen vertretbaren Bereich von 100 pro Jahr“, so Reifart. Aus diesen Zahlen ergebe sich, dass die Senkung des LDL-Cholesterins in der Primärprävention auf $<100 \mathrm{mg} / \mathrm{dl}$ bei Personen mit einem Zehnjahres-Risiko von 5 bis $10 \%$ weiterer Argumente bedarf.

\section{Karotisplaques als Risikoindikator}

Ein wichtiger Risikoindikator, der noch zuverlässiger ist als die Karotis-IntimaMedia-Dicke, ist der Nachweis von Karotisplaques. In einer Metaanalyse von sechs Studien mit 28.000 Patienten war die kardiovaskuläre Ereignisrate bei $\mathrm{Pa}$ tienten mit mittlerem Risikoprofil bei
Nachweis von Plaques um das zwei- bis vierfache erhöht. Die Gesamtereignisrate (Infarkt, Tod, Angina pectoris, Bypass-OP) war in einer anderen Metaanalyse nach 5,4 Jahren bei einem Plaque um $50 \%$, bei zwei und mehr Plaques sogar um $120 \%$ erhöht.

\section{Fazit für die Praxis}

Für Personen mit einem Zehnjahres-Risiko $>20 \%$ ist die Statingabe auch in der Primärprävention unstrittig. Für Personen mit mittlerem Risiko und LDL-C von $100-155 \mathrm{mg} / \mathrm{dl}$ ist dagegen ein Statin nicht sinnvoll, solange keine Karotisplaques nachweisbar sind. „Solche Plaques bedeuten eine deutliche Risikosteigerung, sodass immer unabhängig vom LDL-Cholesterinwert ein Statin anzuraten ist", betonte Reifart. Dies müsse auch für die Krankenkassen einleuchtend sein. Auch sollte man immer wieder daran erinnern, dass eine Korrektur von neun Risikofaktoren $90 \%$ der kardiovaskulären Ereignisse verhindern kann. Dazu gehören neben der Lipidsenkung, der Rauchverzicht, eine optimale Blutzucker- $\left(\mathrm{HbA}_{1 \mathrm{c}}<7 \%\right)$ und Blutdruckeinstellung $(<135 / 85 \mathrm{mmHg})$, Bauchumfang $<88 \mathrm{~cm}$ bei Frauen und $<102 \mathrm{~cm}$ bei Männern, mehr Obst und Gemüse, Bewegung $(2 \times 40 \mathrm{~min} /$ Woche $)$, ein bis zwei Gläser Wein täglich und eine gute soziale Einbindung, also ein Freundes- und Familienkreis.

Dr. med. Peter Stiefelhagen

\title{
Device-Therapie bei chronischer Herzinsuffizienz
}

\begin{abstract}
Eine Device-Therapie bei der chronischen Herzinsuffizienz erfolgt immer auf Grundlage einer optimierten medikamentösen Therapie, wobei sich die Hämodynamik mit dem Device oft erst nach Auftitrierung der Medikamente auf die Zieldosis verbessert. Eine kardiale Resynchronisationstherapie (CRT) ist allerdings nur bei einem Linksschenkelblock sinnvoll. Für Patienten, die nicht für eine CRT geeignet sind, bietet sich die kardiale Kontraktilitätsmodulation (CCM) oder Baroreflex-Aktivierung als Alternative an.
\end{abstract}

\footnotetext{
E in Linksschenkelblock (LSB) bei - herzinsuffizienten Patienten ist nicht nur Folge der linksventrikulären Schädigung, sondern kann auch die Ursache einer Verschlechterung der linksventrikulären Pumpfunktion sein; denn die dadurch hervorgerufene Dyssynchronie führt zu einer Verkürzung der Füllungszeit, zu einer vermehrten Regurgitation an der Mitralklappe, zu einer weiteren Abnahme der Auswurfleistung und einer neurohumoralen Aktivierung. Der ungleiche regionale Stress induziert darüber hinaus eine asymmetrische $\mathrm{Hy}$ pertrophie, interstitielle Fibrose, vermehrte Freisetzung von TNF- $\alpha$ und Apoptose. „Es kommt sogar unter dem Linksschenkelblock zu molekularen Veränderungen im Bereich der Beta-Rezeptoren und Transduktionsproteine", berichtete Prof. Bernd Lemke, Lüdenscheid. Deshalb sei es sinnvoll, die LSB bedingte Dyssynchronisation durch eine CRT zu beseitigen.
}

\section{Resynchronisationstherapie nur bei Linksschenkelblock}

Die einzige Indikation für eine CRT ist ein LSB mit einer QRS-Breite $>120$ msec. Dabei handelt es sich um eine Klasse IEmpfehlung, wobei eine QRS-Dauer $>150 \mathrm{msec}$ das Evidenz-Level A, eine Dauer von 120 bis $150 \mathrm{msec}$ das Level B inne hat. „Nicht LSB-bedingte Verlängerungen der QRS-Dauer stellen heute keine Indikation mehr für eine CRT dar", so Lemke. Deutlich bessere Ergebnisse, sprich höhere Responder-Raten, versprechen die AdaptiveCRT und das MultiPoint-Pacing mit vierpoliger LV-Elektrode. „Mit der adaptiven CRT werden die Resynchronisationsmethode und die AV/VV-Zeiten individuell kontinuierlich und dynamisch mittels eines Algorithmus optimiert und zwar entsprechend dem Überleitungsstatus und Aktivitätsniveau des Patienten, wodurch die Responder-Rate deutlich ansteigt", sagte Lemke. Und dadurch werde auch das klinische Outcome verbessert. Durch die vierpolige Elektrode stieg der Anteil der Responder mit einer Abnahme des endsystolischen Volumens (ESV) um mindestens $15 \%$, von $59 \%$ auf $76 \%$.

\section{Kardiale Kontraktilitätsmodulation als Alternative}

Für Patienten ohne Indikation für eine CRT steht heute die kardiale Kontraktilitätsmodulation (CCM) zur Verfügung. Dabei wird ein „train“ an biphasischen hochenergetischen Impulsen in der absoluten Refraktärphase abgegeben, die keine Erregung und keine Änderung der Aktivierungssequenz zur Folge haben. Das elektrische Feld aktiviert bestimmte Proteinkinasen, womit die Wiederherstellung zellulärer Funktionen ermöglicht wird. Ein anderes innovatives Device für herzinsuffiziente Patienten ist die Baroreflex-Aktivierung. Dabei werden durch Stimulation der Barorezeptoren afferente Signale an das Gehirn gesendet, die eine integrierte Antwort des autonomen Nervensystems mit Hemmung der Sympathikus- und Stimulation der Parasympathikus-Aktivität hervorrufen. „Eine weiteres innovatives Verfahren, für das bisher aber keine klinischen Daten vorliegen, ist die Zwerchfellstimulation", so Lemke.

Dr. med. Peter Stiefelhagen

Quelle: BNK-Jahresabschlusstagung,

28.11.2015 in Leipzig 